\title{
Importance of zero-point energy for crystalline ice phases: A comparison of force fields and density functional theory
}

Cite as: J. Chem. Phys. 150, 234504 (2019); https://doi.org/10.1063/1.5097021

Submitted: 22 March 2019 . Accepted: 21 May 2019. Published Online: 19 June 2019

Soroush Rasti (D), and Jörg Meyer (D)
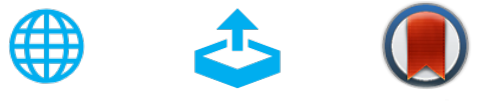

View Online

Export Citation

\section{ARTICLES YOU MAY BE INTERESTED IN}

Is water one liquid or two?

The Journal of Chemical Physics 150, 234503 (2019); https://doi.org/10.1063/1.5096460

Advances in the experimental exploration of water's phase diagram

The Journal of Chemical Physics 150, 060901 (2019); https://doi.org/10.1063/1.5085163

Unsupervised machine learning in atomistic simulations, between predictions and understanding

The Journal of Chemical Physics 150, 150901 (2019); https://doi.org/10.1063/1.5091842

The Journal

of Chemical Physics

The Emerging Investigators Special Collection and Awards Recognizing the excellent work of early career researchers! 


\title{
Importance of zero-point energy for crystalline ice phases: A comparison of force fields and density functional theory
}

\author{
Cite as: J. Chem. Phys. 150, 234504 (2019); doi: 10.1063/1.5097021 \\ Submitted: 22 March 2019 • Accepted: 21 May 2019 • \\ Published Online: 19 June 2019
}

Soroush Rasti $^{\text {a) }}\left(\right.$ D) and Jörg Meyer ${ }^{\text {b) }}($ D

AFFILIATIONS

Gorlaeus Laboratories, Leiden Institute of Chemistry, Leiden University, P.O. Box 9502, 2300 RA Leiden, The Netherlands

Note: This paper is part of a JCP Special Topic on Chemical Physics of Supercooled Water.

a) Electronic mail: s.rasti@lic.leidenuniv.nl

b) Author to whom correspondence should be addressed: j.meyer@chem.leidenuniv.nl

\begin{abstract}
Density functional theory (DFT) including van der Waals (vdW) interactions and accounting for zero-point energy (ZPE) is believed to provide a good description of crystalline ice phases [B. Pamuk et al., Phys. Rev. Lett. 108, 193003 (2012)]. Given the computational cost of DFT, it is not surprising that extensive phonon calculations, which yield the ZPE, have only been done for a limited amount of ice structures. Computationally convenient force fields on the other hand are the method of choice for large systems and/or dynamical simulations, e.g., of supercooled water. Here, we present a systematic comparison for seven hydrogen-ordered crystalline ice phases (Ih, IX, II, XIII, $\mathrm{XIV}, \mathrm{XV}$, and VIII) between many commonly used nonpolarizable force fields and density functionals, including some recently developed meta-GGA functionals and accounting for vdW interactions. Starting from the experimentally determined crystal structures, we perform space-group-constrained structural relaxations. These provide the starting point for highly accurate phonon calculations that yield effectively volume-dependent ZPEs within the quasiharmonic approximation. In particular, when including ZPE, the force fields show a remarkably good performance for equilibrium volumes and cohesive energies superior to many density functionals. A decomposition of the cohesive energies into intramolecular deformation, electrostatic, and vdW contributions quantifies the differences between force fields and DFT. Results for the equilibrium volumes and phase transition pressures for all studied force fields are much more strongly affected by ZPE than all studied density functionals. We track this down to significantly smaller shifts of the O-H-stretch modes and compare with experimental data from Raman spectroscopy.
\end{abstract}

Published under license by AIP Publishing. https://doi.org/10.1063/1.5097021

\section{INTRODUCTION}

Ice is a condensed phase of water that plays an important role in different fields including astrophysics and planetary sciences ${ }^{9-12}$ as well as cryobiology. ${ }^{13}$ It occurs in many different phases due to the large variety of forming different hydrogen bonds between the individual $\mathrm{H}_{2} \mathrm{O}$ molecules. With increasing pressure, these ice phases get more close packed, ${ }^{14}$ which makes their phase diagram and their structures more unusual. ${ }^{15}$ From the 17 known ice phases, ${ }^{16}$ seven are proton ordered and thus have a well-defined crystal structure. These ice phases (ice Ih, ice IX, ice II, ice XIII, ice XIV, ice XV, and ice VIII) capture a wide range of local coordination and thus (hydrogen) bonding scenarios between individual $\mathrm{H}_{2} \mathrm{O}$ molecules in solid water. They are listed together with their space groups and depicted in Table I and Fig. 1, respectively, ordered by increasing pressures at which they form. The geometric structure and relative stability of these different ice phases can be conveniently modeled using small unit cells and periodic boundary conditions in order to compare to available experimental data.

Despite more than 30 years of computer simulations of water, chemical interaction models are still challenged to accurately describe the phase diagram of water ${ }^{17-19}$ due to the complex interplay between hydrogen bonding, ${ }^{20,21}$ van der Waals (vdW), ${ }^{22-2}$ and other nonlocal exchange-correlation effects. ${ }^{18,19,27-29}$ Density 
TABLE I. Bravais lattice, space group, number of water molecules $N$ per unit cell and formation conditions (minimum and maximum pressures $P_{\min }$ and $P_{\max }$, respectively) of the crystalline ice phases considered in this work.

\begin{tabular}{llccc}
\hline \hline Ice & Bravais lattice & Space group & $N$ & $P_{\min }, P_{\max }(\mathrm{GPa})$ \\
\hline Ih & Hexagonal & $P 6_{3} \mathrm{~cm}^{\mathrm{a}}$ & 12 & $0.0,0.2^{\mathrm{b}}$ \\
IX & Tetragonal & $P 4_{1} 2_{1} 2^{\mathrm{c}}$ & 12 & $0.2,0.4^{\mathrm{b}, \mathrm{d}}$ \\
II & Trigonal & $R \overline{3}^{\mathrm{e}}$ & 12 & $0.3,0.5^{\mathrm{b}, \mathrm{f}}$ \\
XIII & Monoclinic & $P 2_{1 / \mathrm{a}} \mathrm{a}^{\mathrm{d}}$ & 28 & $0.5,1.1^{\mathrm{b}}$ \\
XIV & Orthorhombic & $P 2_{1} 2_{1} 2_{1}{ }^{\mathrm{d}}$ & 12 & $1.1,1.3^{\mathrm{b}}$ \\
XV & Triclinic & $P \overline{1}^{\mathrm{f}}$ & 10 & $1.2,1.5^{\mathrm{b}}$ \\
VIII & Tetragonal & $I 4_{1} / a m d^{\mathrm{g}}$ & 8 & $1.5,2.5^{\mathrm{b}, \mathrm{h}}$ \\
\hline \hline
\end{tabular}

${ }^{\mathrm{a}}$ From Ref. 1.

${ }^{\mathrm{b}}$ From Ref. 2 .

${ }^{\mathrm{c}}$ From Ref. 3.

${ }^{\mathrm{d}}$ From Ref. 4.

${ }^{\mathrm{e}}$ From Ref. 5 .

${ }^{\text {f From Ref. } 6 .}$

${ }^{\mathrm{g}}$ From Ref. 7.

${ }^{\mathrm{h}}$ From Ref. 8 .

functional theory (DFT) can capture many of these contributions with varying accuracy for different exchange correlation functionals $^{18,19,24-27}$ and so can force fields (FFs) depending on the sophistication of their parametrization. ${ }^{30}$ Furthermore, vibrational properties can also play an important role, but this has so far been investigated only for a small amount of ice phases. ${ }^{25,26,31-34}$ For example, zero-point energy (ZPE) associated with the lattice vibrations has been found to be responsible for the anomalous volume isotope effect (VIE) of ice $\mathrm{Ih}^{26,35}$ as well as isotope effects for phase transitions.

It is the goal of this study to provide an extensive comparison between off-the-shelve (nonpolarizable) force fields, most of which have been fitted to experimental data for liquid water, and stateof-the-art density functionals for the aforementioned seven protonordered ice phases (Table I and Fig. 1). Given the computational efficiency and good performance in previous studies of ice Ih, II, and III $^{26,32,36}$ compared to path integral molecular dynamics, ${ }^{31}$ lattice dynamics combined with the quasiharmonic approximation (QHA) has been used to obtain the ZPE and accounts for its influence on equilibrium structures and cohesive energies. We find a large effect on structural properties in the case of the force fields and almost none for DFT, which are related to a different description of the $\mathrm{O}-\mathrm{H}$-stretch frequency shifts upon compression and expansion. Likewise, we identify qualitatively different trends for the contributions to the cohesive energies.

This paper is structured as follows: In Sec. II, the theoretical methods and computational details are briefly described. Subsequently, results for the relaxed structures (Sec. III A), the cohesive energies for these structures together with a decomposition into different bonding contributions (Sec. III B) and phase transition pressures (Sec. III C) are presented. This is followed by a detailed analysis of the ZPE (Sec. III D). The paper ends with conclusions and a short outlook on future work in Sec. IV.

\section{METHODOLOGY}

\section{A. Total energy calculations}

The LAMMPS code has been used $^{37}$ in order to calculate total energies, forces, and stress tensors for the $\mathrm{SPC} / \mathrm{E}{ }^{38}$ TIP3P, ${ }^{39}$ TIP4P/2005, ${ }^{40}$ TIP4P/ice, ${ }^{41}$ and $\mathrm{q}-\mathrm{TIP} 4 \mathrm{P} / \mathrm{F}^{42}$ force fields (FFs) that have been parametrized and are commonly used for simulations of water. Harmonic potentials were added to SPC/E, TIP3P, TIP4P/2005, and TIP4P/ice in order to enable intramolecular $\mathrm{OH}$-bond stretching $\left(\omega_{\text {stretch }}=3357 \mathrm{~cm}^{-1}\right)$ and $\mathrm{HOH}$-angle bending $\left(\omega_{\text {bend }}=1610 \mathrm{~cm}^{-1}\right)$ based on experimental data ${ }^{43}$ for the corresponding vibrational modes of liquid water. q-TIP4P/F already describes flexible water molecules by construction. ${ }^{42}$ The LenardJones parts of these force fields have been truncated at a cut-off distance of $9 \AA$. Long-range Coulomb interactions are accounted for via Ewald summation.

DFT calculations at the $\mathrm{LDA}^{45}$ and $\mathrm{GGA}^{46}$ level have been carried out with the FHI-aims package ${ }^{47,48}$ using the standard tight settings. For the latter, pairwise dispersion interactions were added to the PBE exchange-correlation functional ${ }^{46}$ using the TkatchenkoScheffler $(\mathrm{PBE}+\mathrm{TS})^{49}$ as well as the many body dispersion correction $(\mathrm{PBE}+\mathrm{MBD})^{50}$ methods. Calculations at the meta-GGA level (and beyond) were performed with the VASP code $^{51,52}$ using the hard projector-augmented-wave (PAW) potentials ${ }^{53}$ for hydrogen and oxygen included with VASP $^{54}$ together with a plane-wave cutoff energy of $900 \mathrm{eV}$. The SCAN ${ }^{55}$ and SCAN $+\mathrm{rVV}^{5} 0^{56}$ exchangecorrelations functionals have been used, where the latter includes the nonlocal rVV10 van der Waals functional ${ }^{57,58}$ on top of the SCAN meta-GGA. In all cases, a $4 \times 4 \times 4$ Monkhorst-Pack grid $^{59}$ is used for Brillouin zone sampling.

For the force fields, the total energy $U^{\mathrm{FF}}$ can be decomposed according to

$$
U^{\mathrm{FF}}=U^{\mathrm{mol}}+U^{\mathrm{Coul}}+\underbrace{U^{\mathrm{LJ}-\mathrm{r}}+U^{\mathrm{LJ}-\mathrm{a}}}_{U^{\mathrm{LJ}}},
$$

where $U_{\mathrm{mol}}$ is the sum of all intramolecular (stretching and bending) contributions and $U_{\text {Coul }}$ is the electrostatic (Coulomb) energy. $U^{\mathrm{LJ}-\mathrm{r}}$ denote the repulsive and $U^{\mathrm{LJ}-\mathrm{a}}$ denote the attractive part of the Lennard-Jones potential $\left(U^{\mathrm{LJ}}\right)$ that is employed in all of the force fields used in this study in order to account for intermolecular Paulirepulsion and van der Waals (vdW) interactions, respectively. Likewise, for the DFT calculations, it is straightforward to decompose

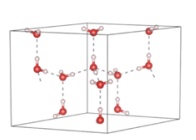

Ice-Ih

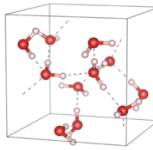

Ice-IX

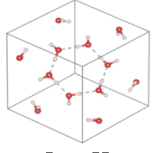

Ice-II

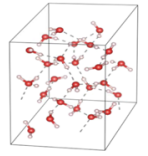

ice-XIII

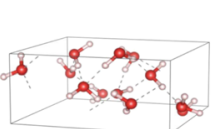

Ice-XIV

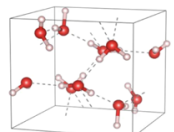

Ice-XV

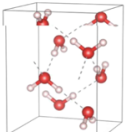

Ice-VIII
FIG. 1. The unit cell of seven proton ordered ice structures. The oxygen and hydrogen atoms are shown in red and white colors, respectively. 
the total energy $U^{\mathrm{DFT}(+\mathrm{vdW})}$ into

$$
U^{\mathrm{DFT}(+\mathrm{vdW})}=U^{\mathrm{kin}+\mathrm{XC}}+U^{\mathrm{Coul}}\left(+U^{\mathrm{vdW}}\right) .
$$

Here, $U_{\text {kin }+\mathrm{XC}}$ is the sum exchange-correlation and kinetic, $U_{\text {Coul }}$ is the Hartree, and $U_{\mathrm{vdW}}$ is the vdW energy.

Data obtained from neutron diffraction experiments $s^{3-7,60}$ have been used in order to generate the initial structures of proton ordered ice phases compiled in Fig. 1 and Table I. As originally suggested by Hamann, ${ }^{1}$ ice Ih has been modeled with a unit cell containing 12 molecules. In order to simultaneously relax the lattice vectors and the internal coordinates of each ice structure while constraining its space group, the algorithm suggested by Pfrommer et al. ${ }^{61}$ has been implemented into the Atomic Simulation Environment. ${ }^{62}$ Using this implementation with the stress tensor and forces obtained from the FF and DFT calculations, space-group constrained equilibrium structures with equilibrium unit cell volume $V_{0}$ could be obtained. A tight (generalized) maximum force threshold of $10^{-4} \mathrm{eV} / \AA ̊$ has been used as convergence criterion for the geometry optimizations. This ensures (vide infra) high-quality phonon calculations. In order to obtain bulk moduli $B_{0}$, energy-volume curves $U(V)$ are fitted to 13 structures within $\pm 4 \%$ of the isotropically contracted and expanded $V_{0}$ using the Rose-Vinet ${ }^{63}$ equation of state (EOS), performing geometry optimizations of the internal coordinates for each of them. Based on the optimized structures, cohesive energies are obtained according to

$$
E_{\text {coh }}=U_{\mathrm{H}_{2} \mathrm{O}}-\frac{1}{N} U_{0, \text { ice }}
$$

in the usual way, where $U_{\mathrm{H}_{2} \mathrm{O}}$ is the total energy of an isolated $\mathrm{H}_{2} \mathrm{O}$ molecule and $U_{0 \text {,ice }}$ is the total energy of the optimized unit cell of the ice phase with $N$ water molecules therein.

\section{B. Inclusion of zero-point energy effects}

The quasiharmonic approximation (QHA) has been used in order to evaluate the Helmholtz free energy,

$$
F(V, T)=U(V)+F_{\text {phonon }}(V, T),
$$

with

$$
F_{\text {phonon }}(V, T)=\underbrace{\frac{1}{2} \sum_{\mathbf{q}, b} \hbar \omega_{\mathbf{q}, b}(V)}_{E_{\mathrm{ZPE}}}+k_{\mathrm{B}} T \sum_{\mathbf{q}, b} \ln \left[1-\exp \left(\frac{-\hbar \omega_{\mathbf{q}, b}(V)}{k_{\mathrm{B}} T}\right)\right] .
$$

Here, $k_{\mathrm{B}}$ is the Boltzmann constant and $\omega_{\mathbf{q}, b}$ is the phonon frequency at wavevector $\mathbf{q}$ for band $b$. The zero-point energy (ZPE) is (equivalently) given by the first moment of the phonon density of states (DOS) $n_{\text {phonon }}$

$$
E_{\mathrm{ZPE}}=F_{\text {phonon }}(V, T=0)=\frac{\hbar}{2} \int_{0}^{\infty} \mathrm{d} \omega \omega n_{\text {phonon }}(\omega),
$$

where $n_{\text {phonon }}(\omega)=\sum_{\mathbf{q}, b} \delta\left(\omega-\omega_{\mathbf{q}, b}\right)$. Because of the volume dependence of the phonon frequencies and the ZPE, the minimum of $F_{\text {phonon }}(V, T)$ with respect to $V$ can be shifted compared to $U(V)$, resulting in equilibrium volumes $V_{0}^{\mathrm{ZPE}}$, bulk moduli $B_{0}^{\mathrm{ZPE}}$, and cohesive energies $E_{\mathrm{coh}}^{\mathrm{ZPE}}$ that account for ZPE effects. These are obtained by calculating phonons for the same 13 structures that have been used for the $U(V)$ curves before. The Parlinski-Li-Kawazoe finite-displacement method ${ }^{64}$ has been employed for the phonon calculations (with displacement of $0.001 \AA$ ) and $F(V, T=0)$-curves fitted employing the Rose-Vinet ${ }^{63}$ EOS, both as implemented in the PHONOPY package. ${ }^{65}$ Exploiting symmetry, the Brillouin zone has been sampled by $30 \times 30 \times 30$ grids for those calculations, which is equal to at least 1456 irreducible $\mathbf{q}$-points for each structure.

\section{Determination of phase-transition pressures}

Transition pressures $P_{\mathrm{A} \rightarrow \mathrm{B}}$, at which an ice phase A goes over into a phase $\mathrm{B}$, are obtained at $T=0$ using three different approximations as follows:

1. The $\Delta$-approximation yields the transition pressure as the negative slope of the common tangent between the $U(V)$-curves,

$$
P_{\mathrm{A} \rightarrow \mathrm{B}}^{\Delta}=-\frac{\Delta U_{0}}{\Delta V_{0}}=-\frac{U_{0, \mathrm{~B}}-U_{0, \mathrm{~A}}}{V_{0, \mathrm{~B}}-V_{0, \mathrm{~A}}},
$$

or the $F(V, T=0)$-curves,

$$
\begin{aligned}
P_{\mathrm{A} \rightarrow \mathrm{B}}^{\Delta, \mathrm{ZPE}} & =-\frac{\Delta F_{0}}{\Delta V_{0}^{\mathrm{ZPE}}} \\
& =-\left.\frac{F\left(V_{0, \mathrm{~B}}^{\mathrm{ZPE}}, T\right)-F\left(V_{0, \mathrm{~A}}^{\mathrm{ZPE}}, T\right)}{V_{0, \mathrm{~B}}^{\mathrm{ZPE}}-V_{0, \mathrm{~A}}^{\mathrm{ZPE}}}\right|_{T=0},
\end{aligned}
$$

of the two ice phases A and B. Obviously, the latter includes ZPE effects.

2. The effect of contraction and expansion can also be included directly in the thermodynamic description by adding $P V$ to $U(V)$ and minimizing the resulting enthalpy with respect to the volume,

$$
H(P)=\min _{V}[U(V)+P V]
$$

The crossing point of $H(P)$ for two ice phases A and B then defines the corresponding transition pressure $P_{\mathrm{A} \rightarrow \mathrm{B}}^{H}$,

$$
H_{A}\left(P_{\mathrm{A} \rightarrow \mathrm{B}}^{H}\right)=H_{B}\left(P_{\mathrm{A} \rightarrow \mathrm{B}}^{H}\right) .
$$

3. Also accounting for the phonon contributions within the QHA, the Gibbs free energy,

$$
G(T, P)=\min _{V}\left[U(V)+F_{\text {phonon }}(V, T)+P V\right],
$$

is calculated in the same fashion $H(P)$ above. The zerotemperature transition pressure $P_{\mathrm{A} \rightarrow \mathrm{B}}^{G}$ is then defined as the pressure where the Gibbs free energies of two ice phases $A$ and $\mathrm{B}$ are equal,

$$
\left.G_{\mathrm{A}}\left(P_{\mathrm{A} \rightarrow \mathrm{B}}^{G}, T\right)\right|_{T=0}=\left.G_{\mathrm{B}}\left(P_{\mathrm{A} \rightarrow \mathrm{B}}^{G}, T\right)\right|_{T=0} .
$$

\section{RESULTS AND DISCUSSIONS}

\section{A. Equilibrium structures}

The detailed results from the structural optimization with all interaction models are provided in the supplementary material. Figure 2 highlights the relative differences of the calculated equi- 


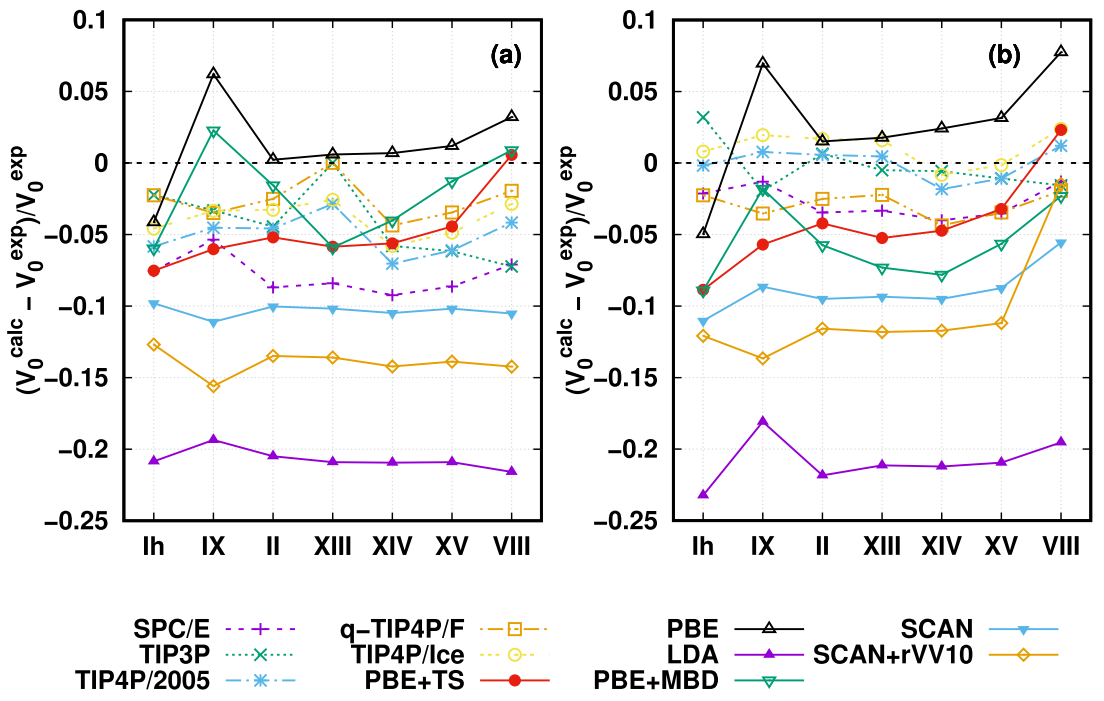

FIG. 2. Relative differences of calculated $V_{0}$ (a) and $V_{0}^{\mathrm{ZPE}}$ (b) from experimental data (black dashed line at 0 ) for the unit cell volumes of the various ice phases. Lines are meant to guide the eye only, with differently colored dashed (solid) lines marking force fields (density functionals) librium volumes with respect to measured data. Without considering ZPE [Fig. 2(a)], q-TIP4P/F shows the best agreement with experiments for all ice phases among the force fields. It yields the smallest average deviation of about $3 \%$, which increases in the order q-TIP4P/F $<$ TIP4P/ice $<$ TIP4P/2005 $<$ TIP3P $<$ SPC/E to almost $8 \%$.

As expected from previous studies for selected ice phases, LDA shows the worst performance among all DFT methods. ${ }^{19}$ The PBEbased results are in good agreement with results from previous calculations: ${ }^{27}$ PBE shows an overall good performance, while including the TS and MBD corrections to account for vdW-interactions improve the PBE structures for high-pressure ice phases. On the other hand, the equilibrium volumes of the low-pressure ice phases (ice Ih in particular) are better described by TIP3P and q-TIP4P/F. The more compact forms of the high-pressure ice phases thus pose a much bigger challenge to the force fields to properly account for molecular deformation, vdW interactions hydrogen bonding networks. On average though, the deviation of the equilibrium volumes for all PBE-based methods is comparable to the TIP4P-family FFs. Quite by contrast, SCAN and (even worse) SCAN+RVV10 show significantly larger deviations from the experimentally determined structures than all force fields.

Figure 2(b) shows the results for the equilibrium volumes including ZPE. Apart from PBE, the volumes for all DFT methods increase very little toward the corresponding experimental data. ZPE-corrected PBE also yields enlarged unit cells, but these now become too large. The ZPE-corrected equilibrium volumes for all FFs increase much more and become significantly closer to the experimental data. Consequently, the average deviations for the latter become less than 3\% and thus outperform all DFT methods. The importance of individual phonon modes for this result will be analyzed in more detail in Sec. III D.

\section{B. Cohesive energies}

The cohesive energy per $\mathrm{H}_{2} \mathrm{O}$ molecule allows us to characterize the relative stability of the different ice phases. Experimental data are available ice Ih, IX, II, and VIII from Whalley ${ }^{66}$ without and with ZPE. ZPE is excluded from the latter in a linear fashion, and ice IX, II, and VIII are less stable than ice Ih by 5,1 , and $33 \mathrm{meV} / \mathrm{H}_{2} \mathrm{O}$, respectively. These data are shown together with the results of the calculations from this work without (with) ZPE in Figs. 3(a) and 3(b). The sequence of increasingly compressed ice structures listed in Table I is, therefore, expected to decrease in stability and thus yield decreasing cohesive energies.

Without ZPE [see Fig. 3(a)], all methods correctly predict ice Ih (ice VIII) to be most (least) stable. Only the SCAN functional yields the relative cohesive energies in outstanding quantitative agreement with experiments. It is, therefore, the only method that is able to predict that ice II is more stable than ice IX as has been observed before by Sun $e$ e $a l .{ }^{29}$ In addition to SCAN, the near degeneracy between ice Ih and ice II is only captured correctly with DFT functionals that explicitly account for vdW interactions $(\mathrm{PBE}+\mathrm{TS}, \mathrm{PBE}+\mathrm{MBD}$, and SCAN+rVV10). Still, all DFT methods functionals overbind the structures by more than $50 \mathrm{meV}$ per water molecule with LDA being significantly further away. Absolute cohesive energies are on average much better described by all the FFs, except for TIP4P/ice, which surprisingly shows the largest offset with respect to the experimental data. The relative stability can be problematic (in particular for TIP3P). Like for the equilibrium volumes, q-TIP4P/F performs best overall by predicting even the absolute cohesive energies very accurately.

Including ZPE reduces the cohesive energy in all calculations [see Fig. 3(b)] as expected according to the ZPE correction employed by Whalley. ${ }^{66}$ Consequently, the superiority of the FFs for the prediction of the absolute cohesive energies does not change. q-TIP4P/F remains the best choice among the FFs, and TIP4P/ice remains the worst. The prediction of relative stability does not improve or even gets worse for TIP3P. For the DFT methods, LDA gains the biggest improvements due to ZPE but still shows the worst description. The cohesive energies of PBE and PBE+TS improve slightly for ice VIII. For the SCAN functional on the other hand, the inclusion of ZPE very slightly worsens the predicted relative stability of the ice phases. 

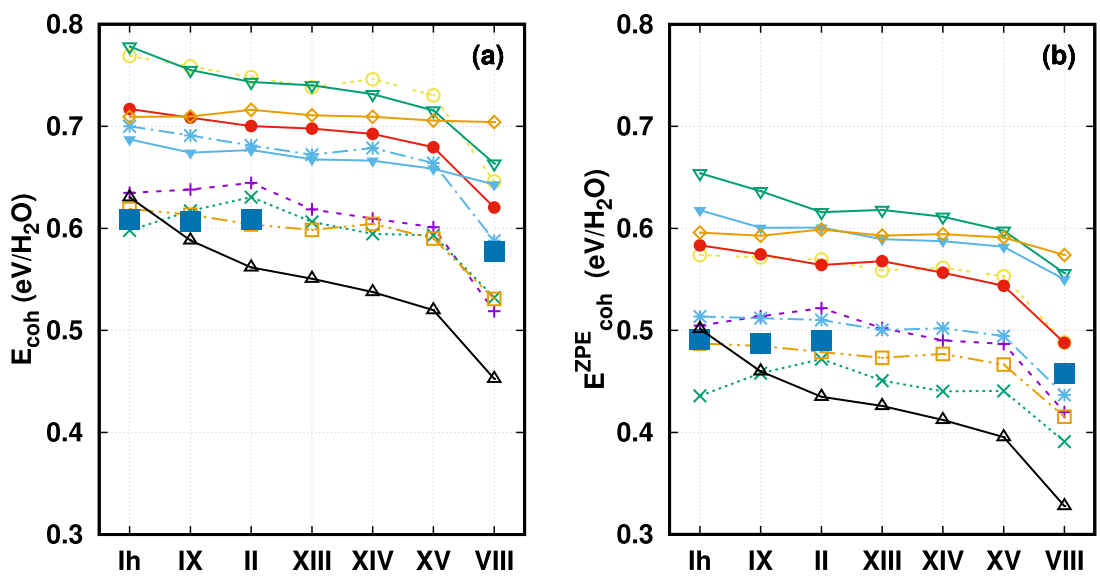

FIG. 3. Cohesive energies per water molecule for the various ice phases without (a) and with (b) account for ZPE. Lines are meant to guide the eye only, with differently colored dashed (solid) lines marking force fields (density functionals). Experimental data from Whalley ${ }^{66}$ are shown by blue squares, without (a) and with (b) ZPE correction suggested as part of that work. Due to the strong overbinding of LDA relative to the experimental reference, results are not shown here but provided in the supplementary material together with all other numerical values.
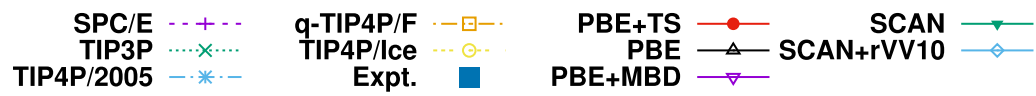

In order to analyze where the differences of the cohesive energies come from, Fig. 4 shows a decomposition into the total-energy contributions described in Sec. II A. As shown by the negative sign of $E_{\mathrm{coh}}^{\mathrm{mol}}+E_{\mathrm{coh}}^{\mathrm{LJ}-\mathrm{r}}$ for the FFs [Fig. 4(a)] and $E_{\mathrm{coh}}^{\mathrm{kin}+\mathrm{xc}}$ for almost all DFT methods [Fig. 4(d)], these contributions decrease the absolute cohesive energy of each ice phase due to structural deformation in the crystal compared to the gas phase. LDA as well as SCAN for ice VIII and PBE+TS are noteworthy exceptions to this trend by yielding positive $E_{\mathrm{coh}}^{\mathrm{kin}+\mathrm{xc}}$. Overall, for both DFT and FFs, the destabilization decreases for the more compact ice phases with increasing pressure.

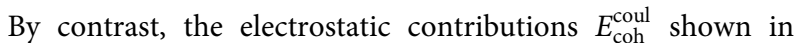
Figs. 4(b) and 4(e) FFs and DFT, respectively, stabilize each ice phase, and the stabilization reduces from ice Ih to ice VIII. For LDA, the magnitude of the stabilization can be up to two times larger than for TIP4P/ice, which has the largest $E_{\text {coh }}^{\text {coul }}$ among the force fields.

Naturally, the contributions to the cohesive energies related to the attractive part of the Lennard-Jones potential $E_{\text {coh }}^{\mathrm{LJ}-\mathrm{a}}$ in case of the FFs [Fig. 4(c)] and van der Waals energies $E_{\text {coh }}^{\mathrm{vdW}}$ for PBE+TS and PBE+MBD [Fig. 4(f)] stabilize each ice phase. The latter two DFT methods, which explicitly separate $U^{\mathrm{vdW}}$, show a monotonously increasing stabilization from ice Ih to ice VIII. In particular, as already discussed by Santra et al., ${ }^{24} E_{\text {coh }}^{\mathrm{vdW}}$ for PBE+TS stabilizes ice VIII about two times more than ice Ih. Figure 4(f) shows that PBE+MBD yields almost the same result. $E_{\text {coh }}^{\mathrm{LJ}-\mathrm{a}}$ for the FFs is of very similar magnitude but, quite by contrast, does not show such a monotonous trend. This is mirrored by the fact that the Pauli repulsion, which is described by the repulsive part of the LennardJones potential, does not decrease monotonously when going from ice Ih to ice VIII as shown in Fig. 5 for TIP4P/2005 as a representative example. Inspired by the analysis of Santra et al., Fig. 5(a) shows $U^{\mathrm{LJ}}$ as a function of contributing pairs in growing neighbor shells that can be characterized by maximum oxygenoxygen distances $R$. For TIP4P/2005 and equivalently for all the other FFs, the first neighbor shell at $\leq 3 \AA$ is in the repulsive regime of the Lennard-Jones potentials used for the FFs (e.g., $\sigma=3.1668 \AA$ for TIP4P $/ 2005^{41}$ ). Only subsequent neighbor shells then accumulate attractive contributions $U^{\mathrm{LJ}-\mathrm{a}}$ to $U^{\mathrm{LJ}}$. Compared to PBE+TS [Fig. 5(b)] (for which all neighbors yield attractive contributions to $U^{\mathrm{vdW}}$ by construction), the $U^{\mathrm{LJ}-\mathrm{a}}$ contributions to TIP4P/2005 are much smaller at comparable distances. Altogether, since the parameters of $U^{\mathrm{LJ}}$ of the FFs have been fitted without separating $U^{\mathrm{LJ}-\mathrm{r}}$ and $U^{\mathrm{LJ}-\mathrm{a}}$, it is not surprising that $E_{\mathrm{coh}}^{\mathrm{LJ}-\mathrm{a}}$ and $E_{\mathrm{coh}}^{\mathrm{vdW}}$ show different trends.

\section{Phase transition pressures}

Since phase transition pressures for two ice phases A and B are generally influenced both by structural and energetic effects, it is interesting to calculate the latter using the different techniques described in Sec. II C and compare with experimental reference values. ${ }^{2,4,6,8}$ Figure 6 shows the results for the $\Delta$-approximation. Now, values are shown when the transition pressure turn out to be negative. As observed by Santra et al. ${ }^{27}$ before, without taking ZPE into account [Fig. 6(a)], PBE largely overestimates $P_{\mathrm{A} \rightarrow \mathrm{B}}^{\Delta}$ but can be significantly improved by accounting for vdW interactions in $\mathrm{PBE}+\mathrm{MBD}$ and (even more) PBE+TS. LDA benefits from error canceling for both the equilibrium volumes and cohesive energies and does slightly better than PBE but not as good PBE+MBD. The SCAN functional performs even better than PBE+TS, and SCAN+rVV10 (likewise with account for vdW) yields the best results. Due to the problems with the relative stability of the ice phases discussed in Sec. III B, only q-TIP4P/F, again the best among the FFs, yielding meaningful results for all transitions which are comparable to PBE+TS. Including ZPE [Fig. 6(b)] does not change most of the DFT results except for SCAN+rVV10, which now yields positive $P_{\mathrm{A} \rightarrow \mathrm{B}}^{\Delta, \mathrm{ZPE}}$ 

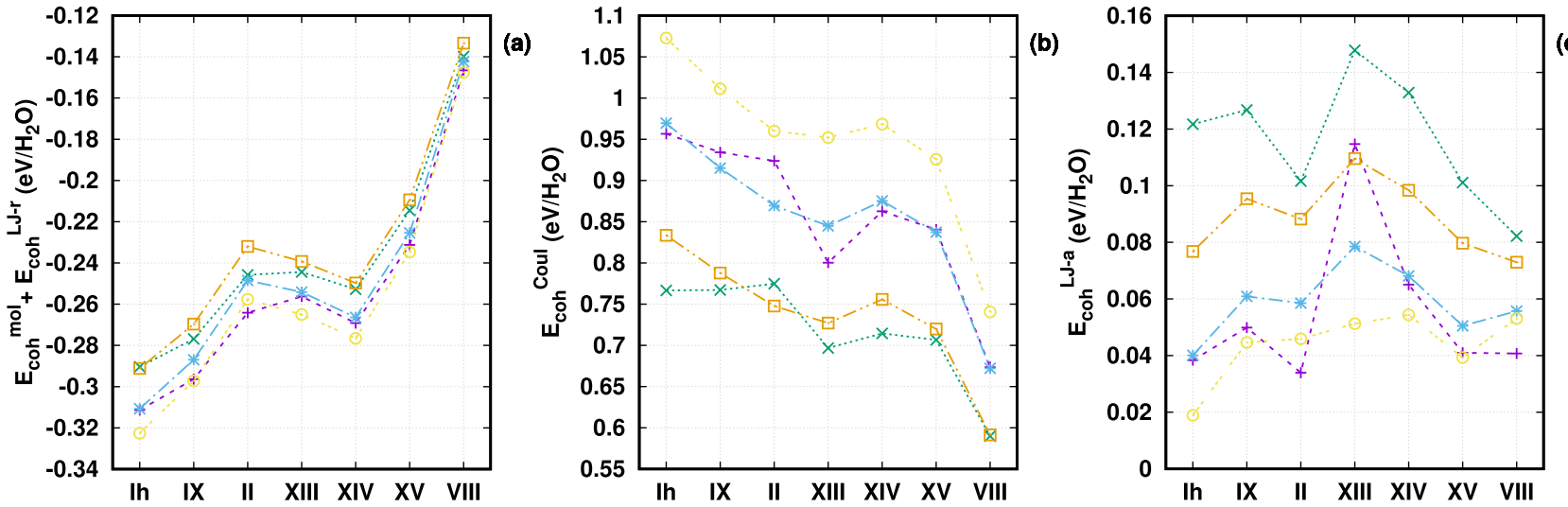

(c)

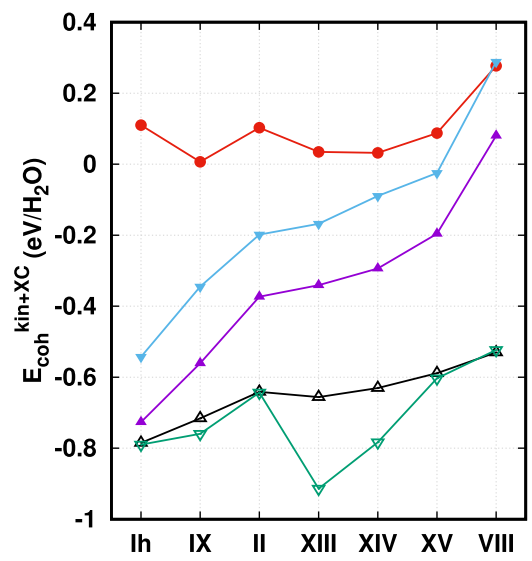

(d)

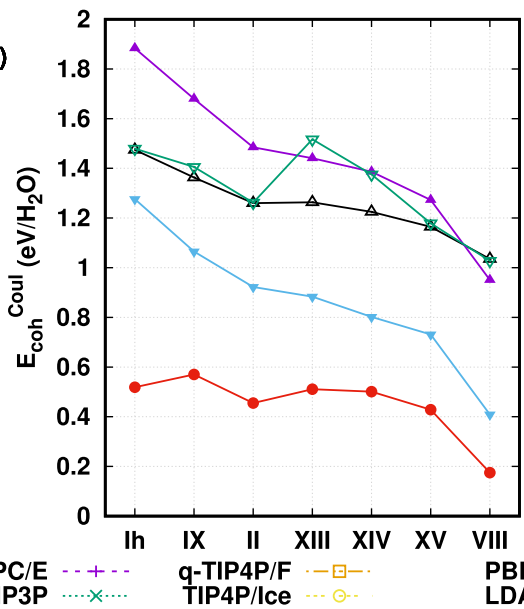

(e)

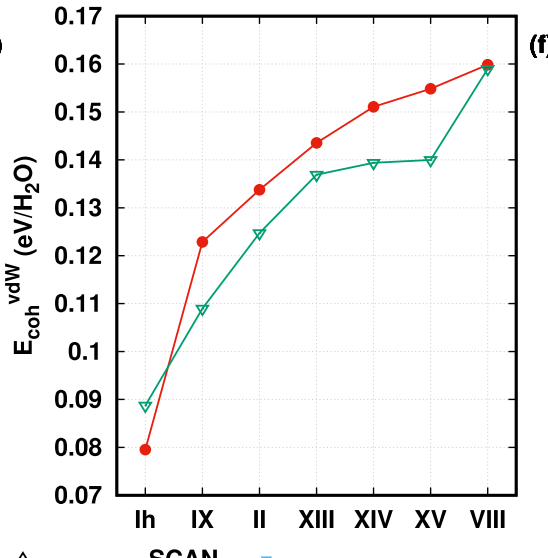
TIP4P/2005 PBE+TS

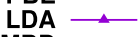

SCAN $\longrightarrow$

FIG. 4. Decomposition of cohesive energy without ZPE for the various ice phases [see 3(a)] into contributions to the total energies for FFs [(a)-(c); see Eq. (1)] and DFT [(d)-(f); see Eq. (2)]. Lines are meant to guide the eye only, with differently colored dashed (solid) lines marking force fields (density functionals).

for all transitions. The same happens for all force fields apart from SPC/E. Their agreement with the available experimental data is not as good as for SCAN+rVV10 but still much better than LDA, SCAN, and all PBE-based methods.

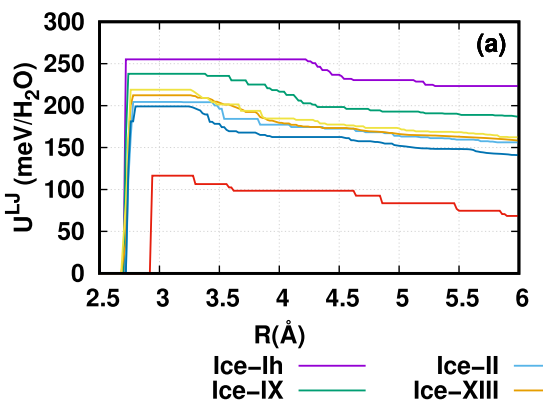

Transition pressures obtained based on the enthalpy and Gibbs free energy, $P_{\mathrm{A} \rightarrow \mathrm{B}}^{H}$ and $P_{\mathrm{A} \rightarrow \mathrm{B}}^{G}$ shown in Figs. $7(\mathrm{a})$ and 7(b), respectively, which both include ZPE, follow the same qualitative trends. However, in case of $P_{\mathrm{A} \rightarrow \mathrm{B}}^{H}$, more DFT methods and FFs than for

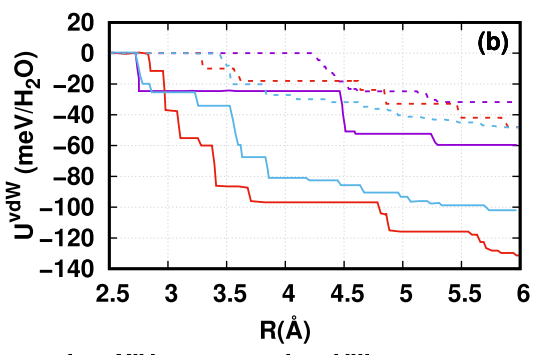

Ice-XIV

Ice-VIII

FIG. 5. The solid lines show the accumulated the Lennard-Jones potential $U^{L L}$ for TIP4P/2005 [this work, (a)] and van der Waals energies $U^{\text {vdW }}$ for PBE+TS [data from Santra et al. ${ }^{24}$ (b)], when more and more pairs contribute with increasing oxygen-oxygen distance $R$. Different ice phases are shown by the differently colored lines. In panel (b), the attractive part of the Lennard-Jones potential $U^{L J-a}$ for TIP4P/2005 is indicated by dashed lines for comparison. 


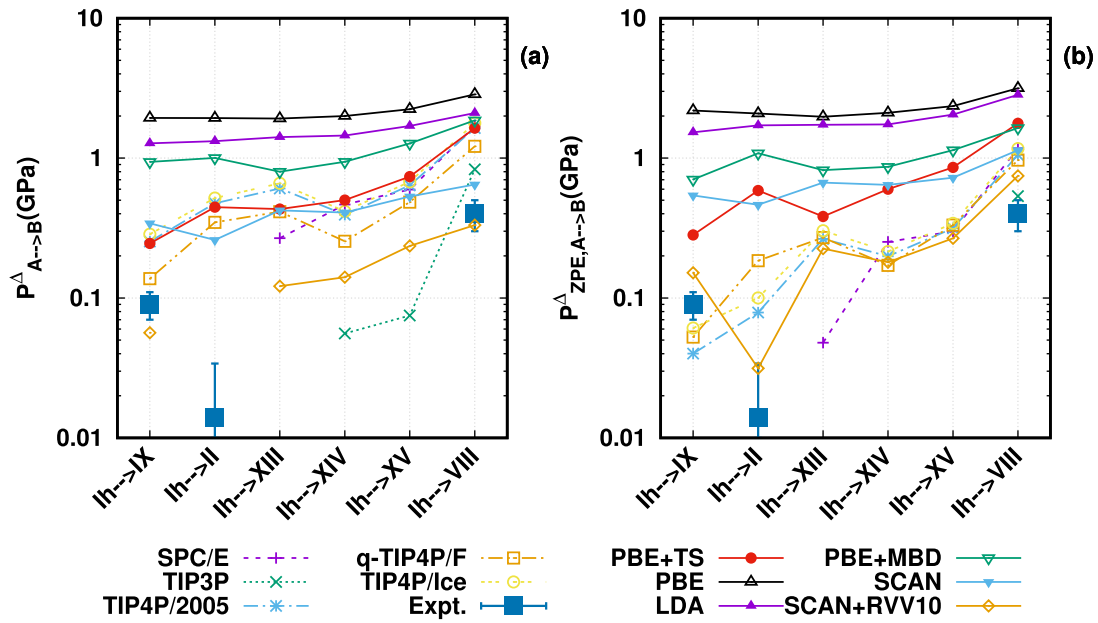

FIG. 6. Phase transition pressures at which ice Ih goes over into the other six ice phases (IX, II, XIII, XIV, and VIII) considered in this work as obtained by the $\Delta$-approximation (a) without and (b) ZPE [see Eqs. (7) and (8) in Sec. II C, respectively]. Lines are meant to guide the eye only, with differently colored dashed (solid) lines marking force fields (density functionals). The blue filled squares show experimental data extrapolated to the zero temperature including error bars where available as given by Whalley.

$P_{\mathrm{A} \rightarrow \mathrm{B}}^{\Delta, \mathrm{ZPE}}$ failed to predict a positive values or simply yield results that are out of the range plotted in Fig. 7(a). Results based on the Gibbs free energy on the other hand are comparable to the ZPE-corrected $\Delta$-approximation. Furthermore, those results for $P_{\mathrm{A} \rightarrow \mathrm{B}}^{G}$ shown in Fig. 7(b) are only mildly affected by temperature, i.e., the change in the worst case by about $0.08 \mathrm{GPa}$ at $T=200 \mathrm{~K}$.

\section{Analysis of zero-point energy effects}

As observed in Secs. III A-III C, ZPE has much more pronounced effects on the results of FF compared to DFT calculations. These effects originate from the influence of the QHA (see Sec. II B) on the equation of state for the different ice phases, i.e., phonon frequencies must change significantly differently for these two families of interaction potentials when compressing or expanding the unit cell. Focusing on ice II, Fig. 8 illustrates the reason for these differences by taking TIP4P/ice and PBE+TS as representative examples for the respective families. The other family members show the same qualitative trend for ice II and also the other ice structures.

The phonon DOS for TIP4P/ice [Fig. 8(a)] shows strong shifts toward higher (lower) for the crystal (frequency interval from $0 \mathrm{~cm}^{-1}$ to $\left.500 \mathrm{~cm}^{-1}\right)$ and librational $\left(500 \mathrm{~cm}^{-1}-1500 \mathrm{~cm}^{-1}\right)$ modes upon compression (expansion). The molecular bending $\left(1500 \mathrm{~cm}^{-1}-2000 \mathrm{~cm}^{-1}\right)$ and stretching modes $\left(2000 \mathrm{~cm}^{-1}-\right.$ $3500 \mathrm{~cm}^{-1}$ ) on the other hand are hardly affected. Consequently, the first moment of the phonon DOSs [i.e., $E_{\mathrm{ZP}, \mathrm{TIP} 4 \mathrm{P} / \text { ice }}(V)$; see Eq. (6)] is monotonously increasing (decreasing) for $V<V_{0}\left(V>V_{0}\right)$, and thus the minimum of $F_{\mathrm{TIP} 4 \mathrm{P} / \mathrm{ice}}(V, T=0)$ (see Ref. 5 ) shifts to the right, as shown in Fig. 8(b). This is in good agreement with the microscopic Grüneisen parameters $\left(\gamma_{i}=-\frac{V}{\omega_{i}} \frac{\partial \omega_{i}}{\partial V}\right)$ that Ramírez et al. ${ }^{32}$ have calculated for ice II using the q-TIP4P/F model.

For PBE+TS, the upward (downward) shifts of the crystal and librational modes due to compression (expansion) are almost the same as for TIP4P/ice [Fig. 8(c)]. However, only the bending modes remain unaffected, whereas the stretching modes shift in the

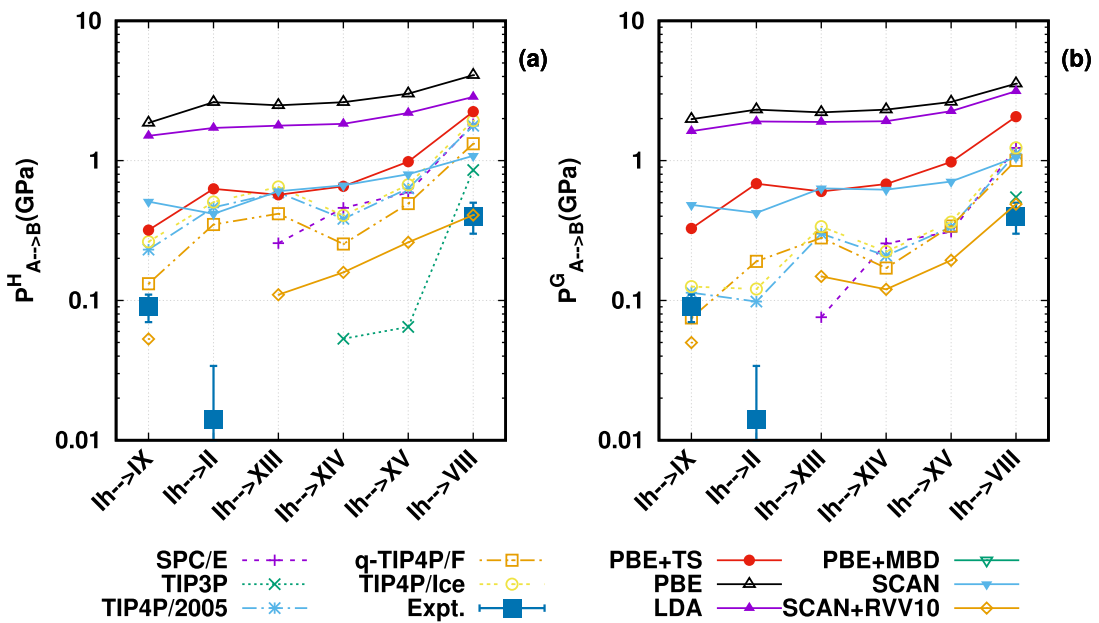

FIG. 7. Same as Fig. 6, but for phase transition pressures obtained from (a) enthalpy and (b) Gibbs free energy [see Eqs. (10) and (12) in Sec. II C, respectively] 

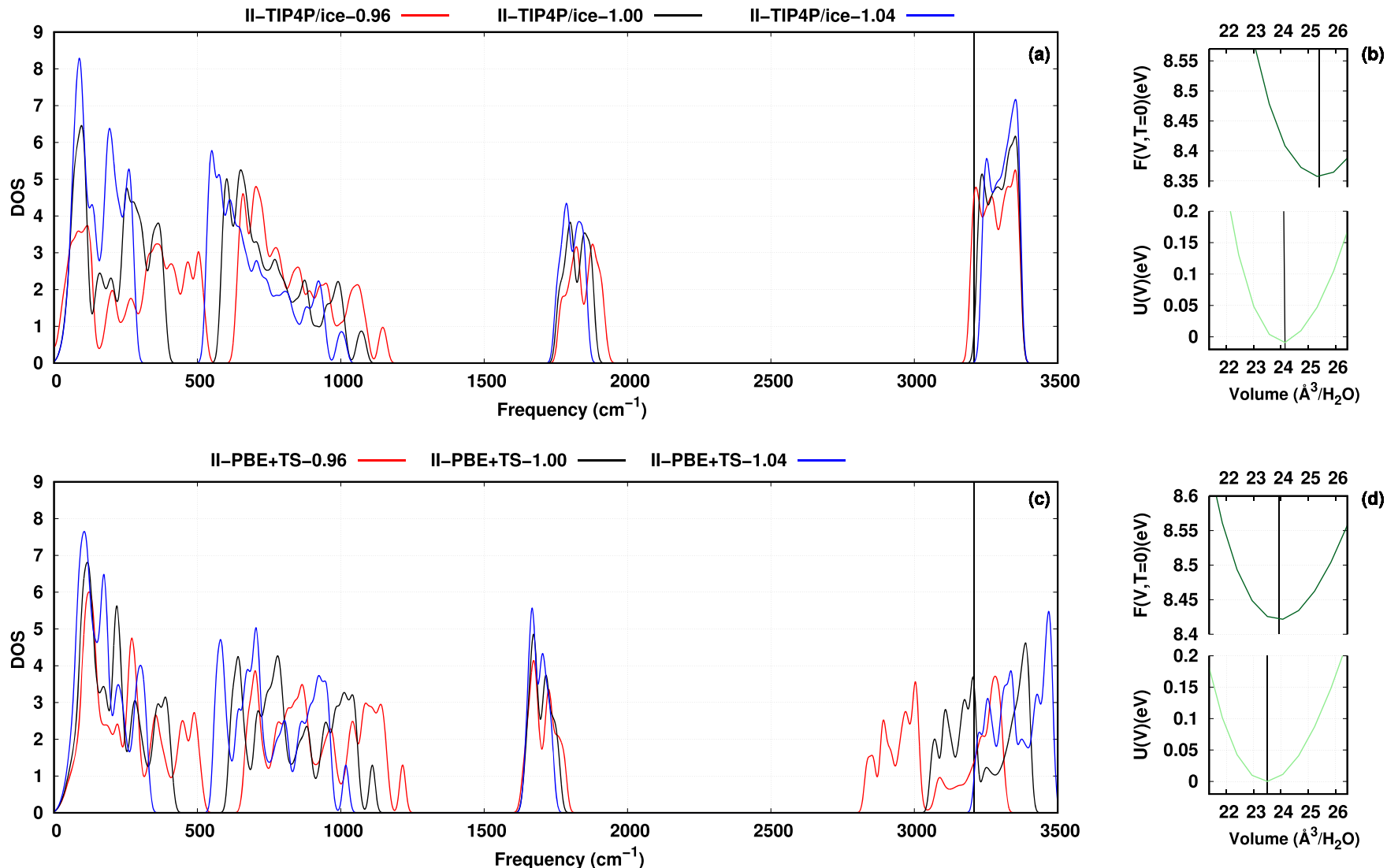

FIG. 8. Phonon densities of states for ice II obtained with TIP4P/ice (a) and PBE + TS (c). Black, red, and blue lines show the phonon DOSs at the corresponding equilibrium volumes $\left(V_{0}\right)$ as well as isotropically compressed $\left(0.96 \cdot V_{0}\right)$ and expanded $\left(1.04 \cdot V_{0}\right)$ structures, respectively. Experimental data for the stretching frequencies are indicated by the black vertical lines. Internal energy $U(V)$ (dark green line) and Helmholtz free energy $F(V, T=0)$ (light green line) are plotted for TIP4P/ice (b) and PBE $+T S(d)$ using $U\left(V_{0, \text { TPAPlice }}\right)$ and $U\left(V_{0, \mathrm{PBE}+\mathrm{TS}}\right)$ as energy zeros, respectively. $V_{0, \mathrm{TP} 4 P \text { Pice }}\left(V_{0, \mathrm{TPP} P \text { Plice }}^{\mathrm{ZPE}}\right)$ and $V_{0, \mathrm{PBE}+\mathrm{TS}}\left(V_{0, \mathrm{PBE}+\mathrm{TS}}^{\mathrm{ZPE}}\right)$ are the corresponding equilibrium volumes without (with) taking ZPE into account, which are indicated by black vertical lines.

exact opposite way. This almost compensates the effect of the lowfrequency modes on the first moment of the phonon DOS so that $E_{\mathrm{ZP}, \mathrm{PBE}+\mathrm{TS}}(V)$ is almost constant and the minima of $U_{\mathrm{PBE}+\mathrm{TS}}(V)$ and $F_{\mathrm{PBE}+\mathrm{TS}}(V, T=0), V_{0, \mathrm{PBE}+\mathrm{TS}}$, and $\left(V_{0, \mathrm{PBE}+\mathrm{TS}}^{\mathrm{ZPE}}\right)$, practically coincide as shown in Fig. 8(d).

According to the Raman spectra measured by MincevaSukarova, Sherman, and Wilkinson, ${ }^{67}$ the change of O-H stretch frequencies with pressure $\frac{\partial v}{\partial P}$ is about $80 \mathrm{~cm}^{-1} / \mathrm{GPa}$ for most crystalline ice phases for a wide range of temperatures between 250 and $0 \mathrm{~K}$. Given a bulk modulus $B_{0}$ between 12 and $16.5 \mathrm{GPa}$ in this temperature range for ice $\mathrm{II}^{68}{ }^{68}$ this allows us to estimate the expected frequency change $\Delta v \approx \frac{-B_{0}}{V_{0}} \frac{\partial v}{\partial P} \Delta V_{0} \approx \pm 38$ to $53 \mathrm{~cm}^{-1}$ for the volume change $\Delta V_{0}= \pm 0.04 \cdot V_{0}$ considered in Fig. 8 . The average frequency shifts according to the data presented in this figure are $10 \mathrm{~cm}^{-1}$ and $116 \mathrm{~cm}^{-1}$ for TIP4P/ice and PBE + TS, respectively, thus revealing slightly larger relative errors for the FF considering the fact that the simulations have been carried out for $0 \mathrm{~K}$. The better description of the equilibrium volumes by the FFs thus appears to be fortuitous error canceling. This is consistent with the failure of
q-TIP4P/F to describe the anomalous volume isotope effect for ice $\mathrm{Ih}^{26}$ as well as isotope dependence of the ice XI-ice Ih phase transition temperature, ${ }^{36}$ which, however, is also quite challenging to be modeled correctly by first-principles-based techniques.

In order to analyze how much harmonic potential used together with TIP4P/ice for the intramolecular O-H-bond (see Sec. II A) affects the results shown in Fig. 8, we turn to q-TIP4P/F, where these bonds are described by a Morse potential. Approximating the latter by a second-order Taylor expansion and keeping all other parameters unchanged (force field labeled q-TIP4P/F-h), we have recalculated the phonon DOS for ice II. The results are shown in Fig. 9(a) in comparison with conventional q-TIP4P/F shown in Fig. 9(b). As expected by construction, only the $\mathrm{O}-\mathrm{H}$ stretching modes in the phonon DOS are different. The harmonic potential in q-TIP4P/F-h yields much more rigid $\mathrm{O}-\mathrm{H}$ bonds than the Morse potential in q-TIP4P/F as evidenced by their position and much smaller shift upon compression and expansion. In fact, for $\mathrm{q}-\mathrm{TIP} 4 \mathrm{P} / \mathrm{F}$, the corresponding average frequency shifts of $87 \mathrm{~cm}^{-1}\left(53 \mathrm{~cm}^{-1}\right)$ for $4 \%$ compression ( $4 \%$ expansion) fit very nicely to the aforementioned 

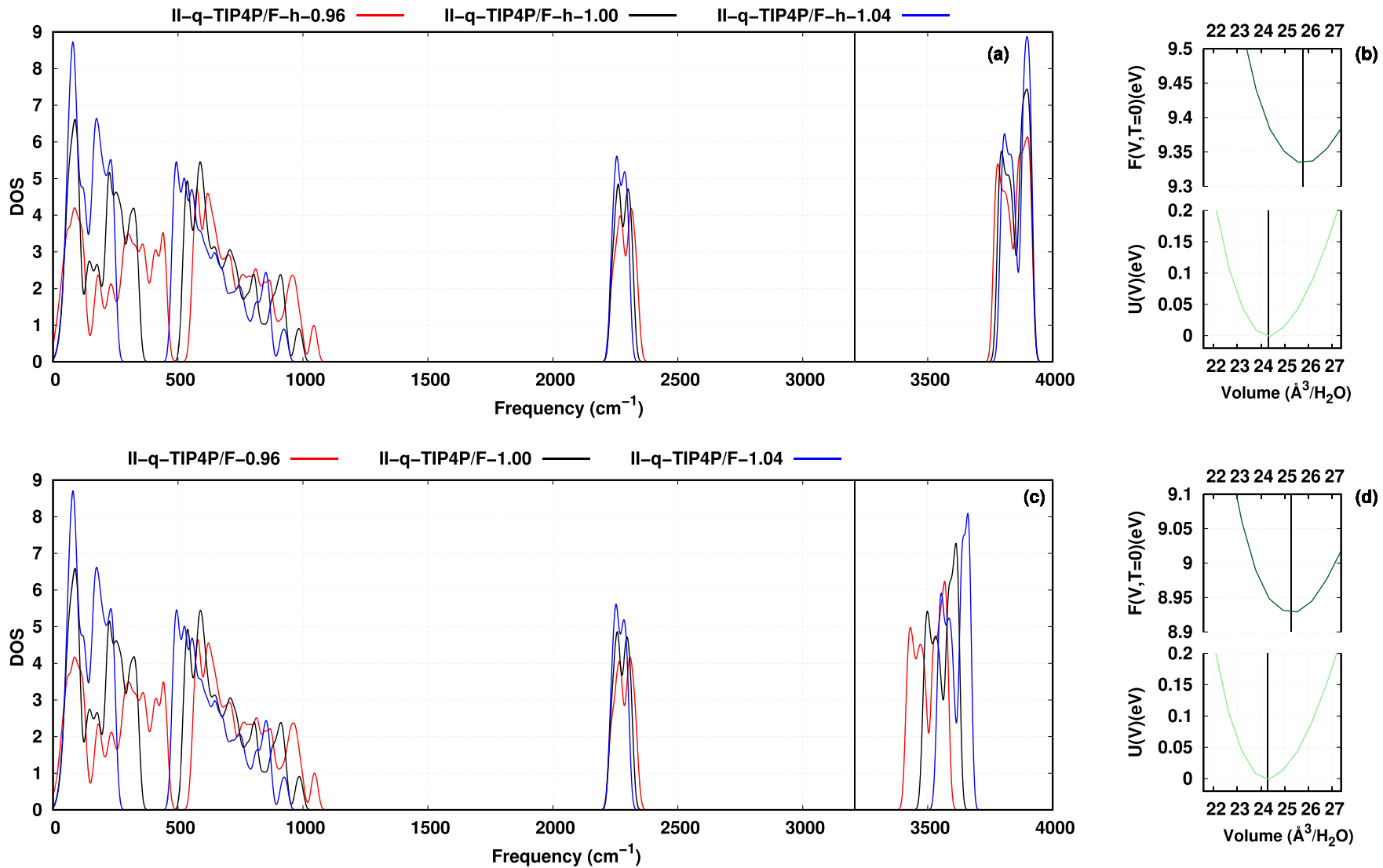

FIG. 9. Same as Fig. 8, but for q-TIP4P/F-h [(a) and (b)] and q-TIP4P/F [(c) and (d)]. In q-TIP4P/F-h, the Morse potential describing the intramolecular OH bond has been replaced by the harmonic potential that is identical to the Morse potential up to second order.

values suggested by the Raman experiments. The strong influence of the Morse potential can be understood by considering its second derivative with respect to the $\mathrm{O}-\mathrm{H}$ bond distance $r^{\mathrm{OH}}$,

$$
\frac{\partial^{2} V^{\mathrm{Morse}}}{\partial r_{\mathrm{OH}}^{2}}=2 D \alpha^{2} e^{-\alpha \Delta r^{\mathrm{OH}}}\left(2 e^{-\alpha \Delta r^{\mathrm{OH}}}-1\right),
$$

where $D$ is the well depth, $\alpha$ is the width, and $\Delta r^{\mathrm{OH}}=r^{\mathrm{OH}}-r_{0}^{\mathrm{OH}}$ is the deviation from the equilibrium $\mathrm{O}-\mathrm{H}$ bond length $r_{0}^{\mathrm{OH}}$ for a single $\mathrm{H}_{2} \mathrm{O}$ molecule. Despite the small changes of the latter in the ice crystal (i.e., $0<\Delta r{ }^{\mathrm{OH}} \ll 1$ ), the exponential terms in Eq. (13) show that vibrational frequencies are very sensitive to $\Delta r^{\mathrm{OH}}$. Quite by contrast, the second derivative of the harmonic potential is constant, i.e., completely unaffected by $\Delta r \mathrm{OH}$.

\section{CONCLUSION}

We have performed a comprehensive study on seven crystalline (proton-ordered) ice phases with a wide range of DFT functional, including the recently developed meta-GGAs SCAN and SCAN+RVV10, and commonly used off-the-shelve (nonpolarizable) water force fields. A particular focus has been on accurate phonon calculations within the quasiharmonic approximation, which has been found to be very successful for ice structures, ${ }^{31,32}$ in order to account for zero-point energy effects. Looking at equilibrium volumes, cohesive energies, and phase transition pressure, the force fields show an overall good or even better performance than DFT. q-TIP4P/F is the clear winner among the FFs considered in this study with $5 \%$ error in equilibrium volume and quite accurate cohesive energy and transition pressures, in particular, when ZPE is taken into account. Quite by contrast, the DFT results are much less affected by ZPE. The DFT functionals struggle much more with a simultaneously good description for all these properties as already discussed in earlier studies. $\mathrm{PBE}+\mathrm{MBD}$ deviates from the cohesive energy by more than $100 \mathrm{meV} / \mathrm{H}_{2} \mathrm{O}$ but shows the best agreement with the experimental volumes. ${ }^{27}$ The SCAN functional underestimates the equilibrium volume by $10 \%$ and overestimates the absolute cohesive energy by $60 \mathrm{meV} / \mathrm{H}_{2} \mathrm{O}$ but yields relative cohesive energies and relative equilibrium volumes that are in remarkable agreement with the experiment.

Our decomposition of the cohesive energies reveals that intramolecular deformation is over-compensated by electrostatics and the attractive part of the Lennard-Jones potentials in case of the FFs. The electrostatics are also the dominant and typically even larger bonding contribution for the DFT methods. In case of 
$\mathrm{PBE}+\mathrm{TS}$ and $\mathrm{PBE}+\mathrm{vdW}$, van der Waals interactions stabilize the crystals additionally. While the latter monotonously increase from ice Ih to ice VIII, this is not the case for the attractive part of the Lennard-Jones potentials. Our analysis of phonon DOSs has revealed that the smaller redshift (blueshift) of the $\mathrm{O}-\mathrm{H}$ stretch vibrations upon compression (expansion) of the crystal (i.e., the corresponding Grüneisen parameters) obtained with all FFs compared to all DFT functionals considered here is responsible for the larger effect of ZPE for the FFs. This is in line with previous work for a few ice structures. ${ }^{26,32}$ A comparison to Raman spectra measured as a function of pressure ${ }^{67}$ indicates that neither shifts are accurate when the intramolecular $\mathrm{O}-\mathrm{H}$ stretching is described with a harmonic potential in case of the FFs. We have clearly identified the Morse potential in q-TIP4P/F to yield a significant improvement. Future work with state-of-the polarizable force fields ${ }^{30}$ could provide valuable insights for both the bonding contributions and the vibrational frequency shifts.

\section{SUPPLEMENTARY MATERIAL}

See supplementary material for optimum lattice parameters, cell volumes per water molecule $V_{0}\left(V_{0}^{\mathrm{ZPE}}\right)$, bulk moduli $B_{0}\left(B_{0}^{\mathrm{ZPE}}\right)$, and cohesive energies $E_{\mathrm{coh}}\left(E_{\mathrm{coh}}^{\mathrm{ZPE}}\right)$ without (with) zero-point energy taken into account.

\section{ACKNOWLEDGMENTS}

J.M. gratefully acknowledges financial support from The Netherlands Organization for Scientific Research (NWO) under Vidi Grant No. 723.014.009. We thank B. Kreupeling for useful discussions.

\section{REFERENCES}

'D. R. Hamann, Phys. Rev. B 55, R10157 (1997).

${ }^{2}$ C. G. Salzmann, P. G. Radaelli, B. Slater, and J. L. Finney, Phys. Chem. Chem. Phys. 13, 18468 (2011).

${ }^{3}$ J. D. Londono, W. F. Kuhs, and J. L. Finney, J. Chem. Phys. 98, 4878 (1993).

${ }^{4}$ C. G. Salzmann, P. G. Radaelli, A. Hallbrucker, E. Mayer, and J. L. Finney, Science 311, 1758 (2006).

${ }^{5}$ C. Lobban, J. L. Finney, and W. F. Kuhs, J. Chem. Phys. 117, 3928 (2002)

${ }^{6}$ C. G. Salzmann, P. G. Radaelli, E. Mayer, and J. L. Finney, Phys. Rev. Lett. 103, 105701 (2009)

${ }^{7}$ W. F. Kuhs, J. L. Finney, C. Vettier, and D. V. Bliss, J. Chem. Phys. 81, 3612 (1984).

${ }^{8}$ G. S. Kell and E. Whalley, J. Chem. Phys. 48, 2359 (1968).

${ }^{9}$ J. Poirier, Nature 299, 683 (1982).

${ }^{10} \mathrm{P}$. Jenniskens and D. Blake, Science 265, 753 (1994).

${ }^{11}$ P. Jenniskens, D. Blake, M. Wilson, and A. Pohorille, Astrophys. J. 455, 389 (1995).

${ }^{12}$ B. J. Murray, D. A. Knopf, and A. K. Bertram, Nature 434, 202 (2005).

${ }^{13}$ P. Mehl and P. Boutron, J. Phys. Colloq. 48, C1 (1987).

${ }^{14}$ C. Lee, D. Vanderbilt, K. Laasonen, R. Car, and M. Parrinello, Phys. Rev. Lett. 69, $462(1992)$

${ }^{15}$ N. H. Fletcher, Rep. Prog. Phys. 34, 913 (1971).

${ }^{16}$ T. L. Malkin, B. J. Murray, C. G. Salzmann, V. Molinero, S. J. Pickering, and T. F. Whale, Phys. Chem. Chem. Phys. 17, 60 (2015).

${ }^{17}$ E. Sanz, C. Vega, J. L. F. Abascal, and L. G. MacDowell, Phys. Rev. Lett. 92, 255701 (2004)

${ }^{18}$ Y. Fang, B. Xiao, J. Tao, J. Sun, and J. P. Perdew, Phys. Rev. B 87, 214101 (2013).
${ }^{19}$ M. J. Gillan, D. Alfè, and A. Michaelides, J. Chem. Phys. 144, 130901 (2016).

${ }^{20} \mathrm{~J}$. Li and D. K. Ross, Nature 365, 327 (1993).

${ }^{21}$ S. J. Singer, J.-L. Kuo, T. K. Hirsch, C. Knight, L. Ojamäe, and M. L. Klein, Phys. Rev. Lett. 94, 135701 (2005).

${ }^{22}$ I. Hamada, J. Chem. Phys. 133, 214503 (2010).

${ }^{23}$ B. Kolb and T. Thonhauser, Phys. Rev. B 84, 045116 (2011).

${ }^{24}$ B. Santra, J. Klimeš, D. Alfè, A. Tkatchenko, B. Slater, A. Michaelides, R. Car, and M. Scheffler, Phys. Rev. Lett. 107, 185701 (2011).

${ }^{25}$ É. D. Murray and G. Galli, Phys. Rev. Lett. 108, 105502 (2012).

${ }^{26}$ B. Pamuk, J. M. Soler, R. Ramírez, C. P. Herrero, P. W. Stephens, P. B. Allen, and M.-V. Fernández-Serra, Phys. Rev. Lett. 108, 193003 (2012).

${ }^{27}$ B. Santra, J. Klimeš, A. Tkatchenko, D. Alfè, B. Slater, A. Michaelides, R. Car, and M. Scheffler, J. Chem. Phys. 139, 154702 (2013).

${ }^{28}$ A. M. Reilly and A. Tkatchenko, J. Chem. Phys. 139, 024705 (2013).

${ }^{29}$ J. Sun, R. C. Remsing, Y. Zhang, Z. Sun, A. Ruzsinszky, H. Peng, Z. Yang, A. Paul, U. Waghmare, X. Wu, M. L. Klein, and J. P. Perdew, Nat. Chem. 8, 831 (2016).

${ }^{30}$ G. A. Cisneros, K. T. Wikfeldt, L. Ojamäe, J. Lu, Y. Xu, H. Torabifard, A. P. Bartók, G. Csányi, V. Molinero, and F. Paesani, Chem. Rev. 116, 7501 (2016).

${ }^{31}$ C. P. Herrero and R. Ramírez, J. Chem. Phys. 134, 094510 (2011).

${ }^{32}$ R. Ramírez, N. Neuerburg, M.-V. Fernández-Serra, and C. P. Herrero, J. Chem. Phys. 137, 044502 (2012).

${ }^{33}$ E. A. Engel, B. Monserrat, and R. J. Needs, J. Chem. Phys. 143, 244708 (2015).

${ }^{34}$ E. A. Engel, B. Monserrat, and R. J. Needs, Phys. Rev. X 5, 021033 (2015).

${ }^{35}$ M. A. Salim, S. Y. Willow, and S. Hirata, J. Chem. Phys. 144, 204503 (2016).

${ }^{36}$ B. Pamuk, P. B. Allen, and M.-V. Fernández-Serra, Phys. Rev. B 92, 134105 (2015).

${ }^{37}$ S. Plimpton, J. Comput. Phys. 117, 1 (1995).

${ }^{38}$ H. J. C. Berendsen, J. R. Grigera, and T. P. Straatsma, J. Phys. Chem. 91, 6269 (1987).

${ }^{39}$ W. L. Jorgensen, J. Chandrasekhar, J. D. Madura, R. W. Impey, and M. L. Klein, J. Chem. Phys. 79, 926 (1983).

${ }^{40}$ J. L. F. Abascal and C. Vega, J. Chem. Phys. 123, 234505 (2005).

${ }^{41}$ J. L. F. Abascal, E. Sanz, R. García Fernández, and C. Vega, J. Chem. Phys. 122, 234511 (2005)

${ }^{42}$ S. Habershon, T. E. Markland, and D. E. Manolopoulos, J. Chem. Phys. 131, 024501 (2009).

${ }^{43}$ D. M. Carey and G. M. Korenowski, J. Chem. Phys. 108, 2669 (1998).

${ }^{44}$ D. Frenkel and B. Smit, Understanding Molecular Simulation: From Algorithms to Applications (Academic Press, San Diego, 2002), Vol. 1.

${ }^{45}$ J. P. Perdew and A. Zunger, Phys. Rev. B 23, 5048 (1981).

${ }^{46}$ J. P. Perdew, K. Burke, and M. Ernzerhof, Phys. Rev. Lett. 77, 3865 (1996).

${ }^{47}$ V. Blum, R. Gehrke, F. Hanke, P. Havu, V. Havu, X. Ren, K. Reuter, and M. Scheffler, Comput. Phys. Commun. 180, 2175 (2009).

${ }^{48}$ V. Havu, V. Blum, P. Havu, and M. Scheffler, J. Comput. Phys. 228, 8367 (2009).

${ }^{49}$ A. Tkatchenko and M. Scheffler, Phys. Rev. Lett. 102, 073005 (2009).

${ }^{50}$ A. Tkatchenko, R. A. DiStasio, R. Car, and M. Scheffler, Phys. Rev. Lett. 108, 236402 (2012).

${ }^{51}$ G. Kresse and J. Hafner, Phys. Rev. B 47, 558 (1993).

${ }^{52}$ G. Kresse and J. Furthmüller, Phys. Rev. B 54, 11169 (1996).

${ }^{53}$ P. E. Blöchl, Phys. Rev. B 50, 17953 (1994).

${ }^{54}$ G. Kresse and D. Joubert, Phys. Rev. B 59, 1758 (1999).

${ }^{55}$ J. Sun, A. Ruzsinszky, and J. P. Perdew, Phys. Rev. Lett. 115, 036402 (2015).

${ }^{56}$ H. Peng, Z.-H. Yang, J. P. Perdew, and J. Sun, Phys. Rev. X 6, 041005 (2016).

${ }^{57}$ O. A. Vydrov and T. Van Voorhis, J. Chem. Phys. 133, 244103 (2010).

${ }^{58}$ R. Sabatini, T. Gorni, and S. de Gironcoli, Phys. Rev. B 87, 041108 (2013).

${ }^{59}$ H. J. Monkhorst and J. D. Pack, Phys. Rev. B 13, 5188 (1976).

${ }^{60}$ C. M. B. Line and R. W. Whitworth, J. Chem. Phys. 104, 10008 (1996).

${ }^{61}$ B. G. Pfrommer, M. Côté, S. G. Louie, and M. L. Cohen, J. Comput. Phys. 131, 233 (1997).

${ }^{62}$ A. H. Larsen, J. J. Mortensen, J. Blomqvist, I. E. Castelli, R. Christensen, M. Dułak, J. Friis, M. N. Groves, B. Hammer, C. Hargus, E. D. Hermes, P. C. 
Jennings, P. B. Jensen, J. Kermode, J. R. Kitchin, E. L. Kolsbjerg, J. Kubal, K. Kaasbjerg, S. Lysgaard, J. B. Maronsson, T. Maxson, T. Olsen, L. Pastewka, A. Peterson, C. Rostgaard, J. Schiøtz, O. Schütt, M. Strange, K. S. Thygesen, T. Vegge, L. Vilhelmsen, M. Walter, Z. Zeng, and K. W. Jacobsen, J. Phys.: Condens. Matter 29, 273002 (2017).

${ }^{63}$ P. Vinet, J. R. Smith, J. Ferrante, and J. H. Rose, Phys. Rev. B 35, 1945 (1987).
${ }^{64}$ K. Parlinski, Z. Q. Li, and Y. Kawazoe, Phys. Rev. Lett. 78, 4063 (1997).

${ }^{65} \mathrm{~A}$. Togo and I. Tanaka, Scr. Mater. 108, 1 (2015).

${ }^{66}$ E. Whalley, J. Chem. Phys. 81, 4087 (1984).

${ }^{67}$ B. Minceva-Sukarova, W. F. Sherman, and G. R. Wilkinson, J. Phys. C: Solid State Phys. 17, 5833 (1984).

${ }^{68}$ A. D. Fortes, I. G. Wood, J. P. Brodholt, and L. Vočadlo, J. Chem. Phys. 119, 4567 (2003). 